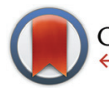

CrossMark

\&lick for updates

Cite this: Analyst, 2016, 141, 827

Received 15th September 2015 Accepted 21st December 2015

DOI: 10.1039/c5an01911g

www.rsc.org/analyst

\section{A ratiometric fluorescent probe for detection of biogenic primary amines with nanomolar sensitivity $\dagger$}

\author{
Suman Mallick, Falguni Chandra and Apurba L. Koner*
}

\begin{abstract}
An ultrasensitive ratiometric fluorescent sensor made of an $\mathrm{N}, \mathrm{N}$-dimethylaminonaphthalene anhydride moiety for detection of aliphatic primary amines is reported. Biogenic amines at nanomolar concentration is detected with the additional ability to discriminate between primary, secondary and tertiary amines by using both UV-Visible and fluorescence spectroscopy.
\end{abstract}

Biogenic amines (BAs) containing at least one aliphatic primary amine group are generated by enzymatic or thermal decarboxylation of amino acids during the storage and processing of food products. ${ }^{1}$ BAs are found in almost all living systems and play crucial roles in varieties of biological processes. ${ }^{2}$ Histamine acts as a neurotransmitter with the addition of an active role in gastric secretion, ${ }^{3}$ whereas spermine and spermidine are involved in cell prolification. ${ }^{4}$ An increased level of BAs in food products causes food poisoning. ${ }^{5}$ Evidently, the accurate detection of BAs in very low concentration is quite important for health and food quality issues. $^{6}$

Several methods to detect BAs and other amine compounds in a qualitative and quantitative manner are already known in the literature. The reported methods include capillary electrophoresis, ${ }^{7}$ various chromatography techniques like high performance liquid chromatography, gas chromatography, enzymatic assay etc. ${ }^{8,9}$ These analytical techniques, though they are very accurate, often require a long time for sample preparation and analysis. In recent years, fluorescence based detection has been widely popular for sensing trace amount of analytes for its superior sensitivity and ease of applicability. Optical methods for detection of small molecules such as amines based on UV-Visible and fluorescence spectroscopic methods are a relatively easier and faster method rather than

Department of Chemistry, Indian Institute of Science Education and Research (IISER), Bhopal By-pass Road, Bhauri, Bhopal-462066, Madhya Pradesh, India. E-mail: akoner@iiserb.ac.in; Fax: +91-755-6692392; Tel: +91-755-6692376 $\dagger$ Electronic supplementary information (ESI) available: Synthetic procedure, UV and fluorescence titrations, CIF file, mass and NMR data. See DOI: 10.1039/ c5an01911g the existing chromatography techniques. The optical detection methods for BAs available are mainly based on fluorescent organic dyes, ${ }^{10-13}$ supramolecular hydrogels,${ }^{14}$ and transition metal complexes. ${ }^{15}$ Until now the probes reported for detecting the amines mainly rely on intensity-based measurements. Generally, they suffer from time taking protocols for preparing the sample before the measurement, lower sensitivity limit and also need a relatively larger amount of amine concentration than the concentration of probe to observe distinguishable signals. ${ }^{16}$ Fluorescence intensity based measurements are susceptible to interference by quenching, scattering, intrinsic auto-fluorescence of samples and are typically inconsistent for being laboratory instrument dependent and can require frequent calibration due to a variety of chemical, optical, or other instrument-related factors. In comparison with intensity based probes, ratiometric probes, which are able to detect fluorescence intensity simultaneously at two different wavelengths, and whose sensing ability dependent on the ratio of the changes observed at two different wavelengths is devoid of instrument dependence, appear to be more potential candidates by overcoming the drawbacks of normal intensity based probes. ${ }^{17-21}$ So, introducing a novel ratiometric probe for detecting BAs has drawn our attention. A comparison of the existing methods and our method for detecting BAs is tabulated in the ESI (see Table S2 $\dagger$ ).

Naphthalimide based intramolecular charge transfer dyes are a very well known class of environmentally sensitive dyes with large Stokes shift, good $\mathrm{pH}$ stability and quantum yield. $^{22,23}$ Due to relatively easy synthetic procedure of 1,8 naphthalimide dyes, they are much more popular compared to other positional isomers of naphthalimide. ${ }^{24}$ These dyes are well studied and used in various fields. But the reports based on 2,3-naphthalimide dyes are relatively less explored. Recently, these 2,3-naphthalimide derivatives have drawn much attention for their versatile use and the development of modern synthetic methods leading to easy synthesis with different functional groups. ${ }^{25}$ This class of dyes have been used widely in various fields such as sensors, ${ }^{26,27}$ intra cellular biomarkers, ${ }^{28}$ fluorescence imaging, ${ }^{23,29}$ DNA photo cleavers, ${ }^{30}$ 
ratiometric $\mathrm{pH}$ sensors,${ }^{31}$ ratiometric DNA detectors, ${ }^{32}$ etc. Our work was motivated by the synthesis and applications of 6-(dimethylamino)naphtho[2,3-c]furan-1,3-dione (ANH) by attaching it in the peptide chain to investigate the proteinprotein interaction. $^{23,28,33}$ Fluorophores containing the anhydride functional group have already been used in several cases in the field of bioconjugate chemistry to tag proteins or peptides for imaging and other studies. ${ }^{22,23,34,35}$ These types of reactions are rapid in nature under ambient conditions and also performable under very dilute conditions $(\mu \mathrm{M})$, suitable for protein labelling experiments, from which we anticipated high reactivity of ANH towards amine groups, paved our way to introduce $\mathrm{ANH}$ as a potential amine sensing candidate.

Herein, we report ANH serving as an ultrasensitive ratiometric optical (UV-Visible, and fluorescent) probe for the detection of aliphatic primary amines, including important biogenic amines with nanomolar sensitivity and also with the additional advantage of the ability to discriminate between primary, secondary and tertiary amines by its differential response properties towards different classes of amines (Fig. 1).

ANH was synthesised following the available protocol in the literature, ${ }^{33,36}$ characterized by ${ }^{1} \mathrm{H}$ NMR, High Resolution Mass Spectrometry (HRMS), and also by single crystal X-ray analysis (see ESI $\uparrow$ for details). The required compound for our spectroscopic study was obtained by multiple recrystallization. To ensure the optical purity of the synthesized probe, we have obtained excitation spectra of ANH at different wavelengths and in two different solvents chloroform and dimethylacetamide (DMA) and compared them with their respective UV spectra (see Fig. S1†). Preliminary tests showed a pronounced effect in both UV-Visible and fluorescence spectra when ANH was subjected to reaction with amines. After that optimization of the experimental conditions for detection of amines results in the following condition, ANH solution $3.68 \mu \mathrm{M}$ in DMA was titrated against different amine analytes to detect amines at micromolar to even sub-micromolar levels both by UV and fluorescence. For detection of amines at nanomolar concen-

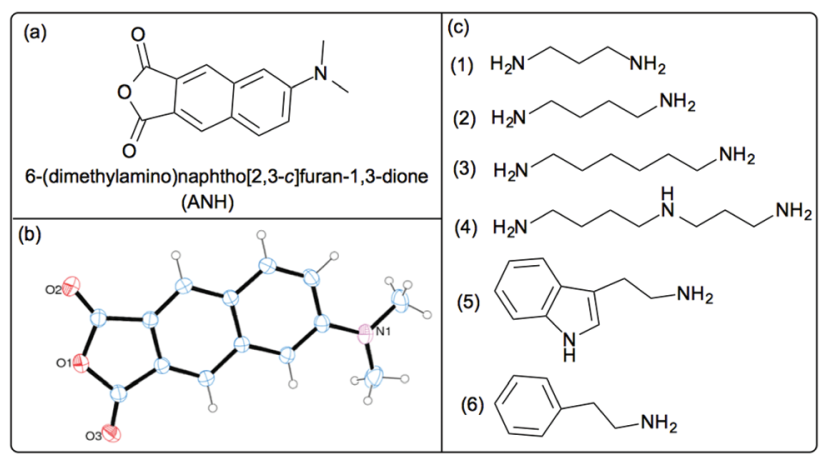

Fig. 1 (a) Structure of ANH, (b) crystal structure of ANH and (c) structure of BAs studied 1 (1,3-propanediamine), 2 (1,4-butanediamine or putrescine), 3 (1,6-hexanediamine), 4 (spermidine), 5 (tryptamine) and 6 (phenethylamine). tration by using fluorescence spectroscopy, we used ANH solution of $0.3 \mu \mathrm{M}$ in DMA for titration against amine analytes.

In this study, we have examined the interaction of $\mathrm{ANH}$ with six different BAs of three different types including 1,3propanediamine, 1,4-butanediamine or putrescine, 1,6-hexanediamine (aliphatic diamines) and spermidine (polyamine), tryptamine, phenethylamine (amine linked to aromatic unit) by UV and fluorescence. We also monitored the interaction of ANH with butylamine (primary amine), diethylamine, diisopropylamine (secondary amine) and triethylamine, diisopropylethylamine (tertiary amine) by UV-Vis and fluorescence spectroscopy. The UV-Visible data for the interaction of $\mathrm{ANH}$ with phenethylamine is depicted in Fig. 2(a). ANH shows a maxima at around $396 \mathrm{~nm}$ in DMA, after interacting with the BAs the intensity of the band centred around $396 \mathrm{~nm}$ starts to decrease and a new band centred around $325 \mathrm{~nm}$ appears with continuous increase in optical density. In each case, we also obtained multiple isosbestic points around 279, 305 and $350 \mathrm{~nm}$, which clearly indicates the transformation of one component (ANH) to another (ANH-amine adduct). For all the amines i.e., primary and secondary the nature of the spectral change in UV is almost the same with a visible colour change from yellow to colourless (see inset of Fig. 2a as a representative example and $\mathrm{ESI}_{\dagger} \dagger$ for others). From Fig. 2(b), it is also evident that the change occurs in a quantitative manner even at sub-micromolar concentration. For primary amines including all the BAs the interaction is always quantitative in nature, but for secondary amines it is not, and for tertiary amines there is almost no change in the UV-Visible spectra (see ESI
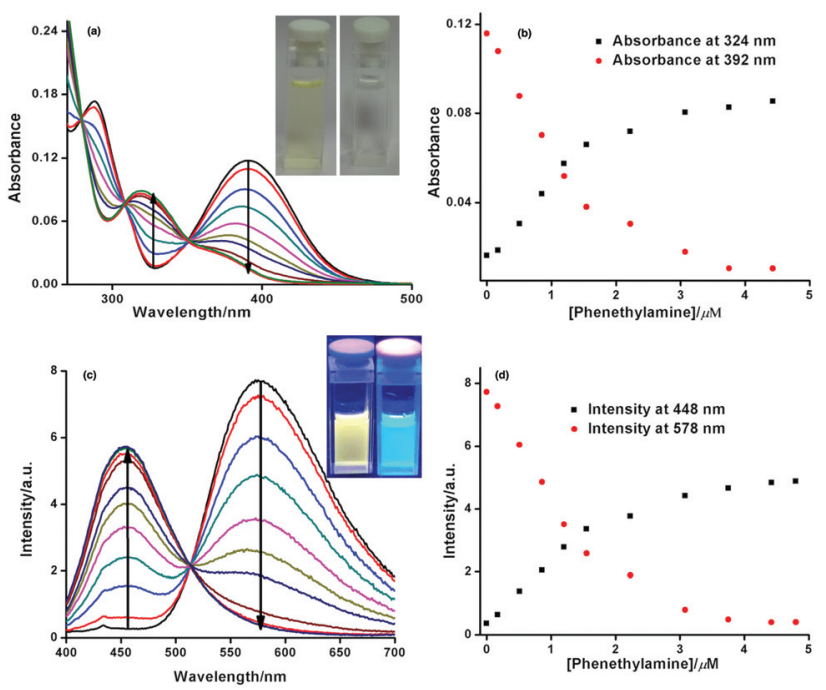

Fig. 2 (a) Titration of ANH with phenethylamine by UV (inset shows digital photograph of ANH solution in DMA before and after the titration). (b) Plot of the change of absorption at two different wavelengths with concentration of phenethylamine. (c) Titration of ANH with phenethylamine using fluorescence by exciting at $385 \mathrm{~nm}$ (inset shows digital fluorescence images of ANH solution in DMA before and after the titration). (d) Plot of the change of intensity at two different wavelengths with concentration of phenethylamine. 
Fig. S2-S16 $†$ for the changes in UV-Visible spectra of ANH after interaction with different amines) that confirms its differential response properties towards different types of amines. We have also tested the effect of other functional groups like primary, secondary, and tertiary alcohols, phenol and aliphatic thiols (see ESI Fig. S16†). These functional groups did not show reactivity towards ANH. Due to a similar response pattern for each case, and offering the opportunity to monitor the changes at two different wavelengths simultaneously also enables ANH to be a new ratiometric UV-Visible probe with excellent sensitivity for the detection of BAs in very low concentration $(\mu \mathrm{M})$ in a quantitative manner. Detection limit for all the BAs by UV-Visible is as low as less than $0.2 \mu \mathrm{M}$ (see ESI $\dagger$ for the detection limits achieved for different amines as analytes by using UV-Visible spectroscopy).

Similarly when the interaction of ANH with different kinds of amines including BAs were subjected to monitoring by fluorescence spectroscopy, it also shows exceptional ratiometric responses and as well as differential responses at micromolar to sub-micromolar concentration towards different types of amines. Initially ANH has a emission maxima around $580 \mathrm{~nm}$ in DMA, after the gradual addition of amines (primary and secondary) the intensity of the emission band with a maxima centred at $578 \mathrm{~nm}$ starts to decrease and a new band with a maxima around $448 \mathrm{~nm}$ starts to generate (see Fig. 2c), with a distinct change in emission colour from orange-yellow to cyan (as shown in the inset of Fig. 2c). So, simultaneous monitoring of the changes of fluorescence intensity observed at $578 \mathrm{~nm}$ and $448 \mathrm{~nm}$ enables us to introduce ANH as a ratiometric fluorescent probe for detection of primary amines including BAs at sub-micromolar concentration in a quantitative manner (see $\mathrm{ESI} \dagger$ for fluorescence emission profile of $\mathrm{ANH}$ by interacting with different BAs and other amines studied). For secondary amines, though the detection limit was in the $\mu \mathrm{M}$ concentration range, the response was not quantitative and there was a very negligible effect on the emission of $\mathrm{ANH}$ when interacted with tertiary amine. By fluorometric detection of different BAs, we always obtain a well-defined isoemissive point centred around $514 \mathrm{~nm}$, indicating the change of one emissive species to another.

Now, keeping in mind the greater sensing ability of fluorescence over other optical sensing methods, we wanted to test the sensing capability of ANH towards different kinds of amines in nanomolar concentration by using fluorescence spectroscopy. For this reason, firstly we performed a fluorescence titration experiment where an $\mathrm{ANH}$ solution of $0.3 \mu \mathrm{M}$ was titrated against gradual addition of phenethylamine (see inset of Fig. 3a). The changes observed in the emission spectra of ANH were very similar as described earlier, and also in a quantitative manner. In this case the detection limit was lowered down, and reached ca. $17 \mathrm{nM}$ (see Fig. 3a). To have a generalized idea about the response of ANH after interacting with different amines at the nanomolar level, we have plotted the data in a bar-chart diagram following the ratiometric response pattern (see Fig. 3b). We have also performed a kinetic experiment to find out the response time for primary,
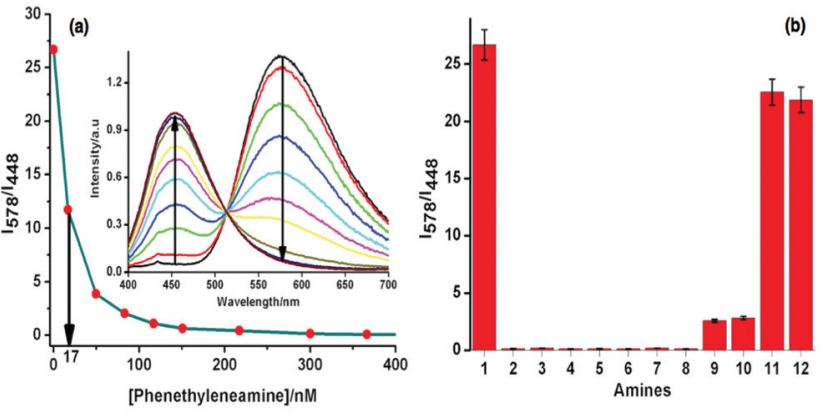

(c)

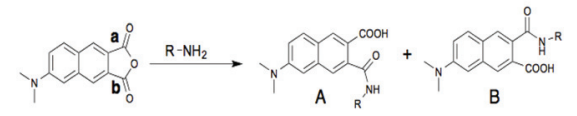

Fig. 3 (a) Titration of ANH with phenethylamine using fluorescence by exciting at $380 \mathrm{~nm}$, results in a detection limit at $17 \mathrm{nM}$, here $I_{578}$ and $I_{448}$ represent their corresponding fluorescence intensity at 578 and $448 \mathrm{~nm}$; the inset shows the gradual changes of fluorescence intensity of ANH by titrating with phenethylamine. (b) $I_{578}$ and $I_{448}$ represent the corresponding fluorescence intensity at 578 and $448 \mathrm{~nm}$ where $50 \mathrm{nM}$ $\mathrm{ANH}$ was treated with $50 \mathrm{nM}$ respective amines 1 : ANH only; 2: tryptamine; 3: phenethylamine; 4: 1,4-butanediamine; 5: 1,6-diaminohexane, 6: 1,3-diaminopropane; 7: butylamine; 8: spermidine; 9: diethylamine; 10: diisopropylamine; 11: triethylamine; 12: diisopropylethylamine. (c) Mechanism of primary amine sensing by $\mathrm{ANH}$.

secondary and tertiary amines (see ESI Fig. S17†). The kinetic experiment shows much faster response of primary amines compared to secondary amines. For the bar-chart diagram experiment, $50 \mathrm{nM}$ ANH was treated with $50 \mathrm{nM}$ respective amines, after 5 minutes fluorescence spectra of the resultant solutions were recorded. From Fig. $3 \mathrm{~b}$ the differential response pattern of $\mathrm{ANH}$ for different types of amines even in nanomolar concentration is very evident. Amines containing at least one primary amine group (entries $2-8$ in Fig. 3 b) show a quantitative response. Secondary amines (entries 9 and 10) show 8-10 fold lower responses as compared to primary amines. But tertiary amines (entries 11 and 12) do not provide any significant response.

For the sensing of amines using $\mathrm{ANH}$, our initial proposition was amine would react with the anhydride unit resulting in two regioisomers by ring opening of the anhydride unit. This will yield one amide bond by nucleophilic addition of the amine group at any of the carbonyl carbons $\mathbf{a}$ and $\mathbf{b}$ and also results in a free carboxyl acid group, as shown schematically in Fig. 3c. Therefore, primary and secondary aliphatic amines satisfy the reaction conditions, but tertiary amines fail to do the same. The spectroscopic analysis also supported our hypothesis, as primary and secondary amines brought notable changes in the UV and fluorescence spectra, but for both the cases the changes were negligible for tertiary amines. Further, to support the sensing mechanism of ANH for different amines, we have collected mass and NMR spectra for the solution of ANH after reacting with butylamine. The results from the mass and NMR experiments also established our hypothesis. After careful analysis of the resulting NMR spectra of 
ANH with butylamine and considering the reactivity of carbonyl carbons positioned at $\mathbf{a}$ and $\mathbf{b}$ in terms of ring opening by amine, we have concluded $\sim 2: 1$ population of $\mathrm{A}: \mathrm{B}$ of the two regioisomers (see $\mathrm{ESI} \dagger$ for the $\mathrm{NMR}$ and mass spectra for detailed analysis, Fig. S18-S20 $\dagger$ ). In the case of sensing diamines, like 1,6-hexanediamine, 1,4-butyldiamine, etc. when the final adducts were subjected to mass spectrometry analysis, it only showed the mass of the monomeric adduct formation (see $\mathrm{ESI} \dagger$ for the mass spectra for detailed analysis, Fig. S21-S23 $\dagger$ ). The reason behind the formation of monomeric adduct is probably that the formation of monomeric adduct is more favoured statistically. The reaction conditions i.e., the very low concentration $(\mu \mathrm{M})$ of $\mathrm{ANH}$ and amine, with a short reaction time (5-10 $\mathrm{min})$ may not be sufficient to form a dimeric adduct with ANH under ambient conditions.

In conclusion, we have shown that ANH can serve as a very efficient ratiometric probe to detect primary amines including BAs rapidly by using UV and fluorescence spectroscopy accompanied by a distinct visible colour change in both the cases and an additional ability to differentiate between different classes of amines. ANH senses the amines by forming a covalent bond between the amine and its anhydride moiety, thus it is irreversible in nature. We have also shown that the sensitivity of ANH towards amines is in the micromolar range by UV-Vis study and in the nanomolar range by fluorescence monitoring, along with that the changes are also quantitative in nature for primary amines including all the BAs studied. In future, we would like to explore the superior sensing property of $\mathrm{ANH}$, so that it can also be utilized as an efficient amine vapour sensor in the solid state by embedding it into a suitable polymer matrix or by the formation of supramolecular assembly employing a suitable host molecule.

S. M. thanks the University Grant Commission (UGC), Government of India, and F. C. thanks IISER Bhopal for their fellowship respectively. The authors would like to thank Dr Debasish Ghorai, IISER Bhopal for his help with the X-ray structure analysis. A. L. K. would like to thank IISER Bhopal for generous financial and infrastructural support.

\section{Notes and references}

1 M. H. S. Santos, Int. J. Food Microbiol., 1996, 29, 213-231.

2 M. Á. Medina, J. L. Urdiales, C. Rodríguez-Caso, F. J. Ramírez and F. Sánchez-Jiménez, Crit. Rev. Biochem. Mol. Biol., 2003, 38, 23-59.

3 H. L. Haas, O. A. Sergeeva and O. Selbach, Physiol. Rev., 2008, 88, 1183-1241.

4 E. Agostinelli, M. P. M. Marques, R. Calheiros, F. P. S. C. Gil, G. Tempera, N. Viceconte, V. Battaglia, S. Grancara and A. Toninello, Amino Acids, 2010, 38, 393403.

5 A. R. Shalaby, Food Res. Int., 1996, 29, 675-690.

6 Y. Salinas, J. V. Ros-Lis, J.-L. Vivancos, R. Martinez-Manez, M. D. Marcos, S. Aucejo, N. Herranz and I. Lorente, Analyst, 2012, 137, 3635-3643.
7 Y.-F. Huang, C.-K. Chiang, Y.-W. Lin, K. Liu, C.-C. Hu, M.-J. Bair and H.-T. Chang, Electrophoresis, 2008, 29, 19421951.

8 A. Önal, Food Chem., 2007, 103, 1475-1486.

9 Z. Song, M. Cai, Y. Zhao, M. Jin and S. Deng, Anal. Methods, 2015, 7, 7436-7442.

10 S. Körsten and G. J. Mohr, Chem. - Eur. J., 2011, 17, 969975.

11 Y. Fu, J. Yao, W. Xu, T. Fan, Q. He, D. Zhu, H. Cao and J. Cheng, Polym. Chem., 2015, 6, 2179-2182.

12 A. R. Longstreet, M. Jo, R. R. Chandler, K. Hanson, N. Zhan, J. J. Hrudka, H. Mattoussi, M. Shatruk and D. T. McQuade, J. Am. Chem. Soc., 2014, 136, 15493-15496.

13 P. G. Sutariya, A. Pandya, A. Lodha and S. K. Menon, Analyst, 2014, 139, 4794-4798.

14 M. Ikeda, T. Yoshii, T. Matsui, T. Tanida, H. Komatsu and I. Hamachi, J. Am. Chem. Soc., 2011, 133, 1670-1673.

15 W. Q. Ong, H. Zhao, C. Sun, J. E. Wu, Z. Wong, S. F. Y. Li, Y. Hong and H. Zeng, Chem. Commun., 2012, 48, 63436345.

16 B. Lee, R. Scopelliti and K. Severin, Chem. Commun., 2011, 47, 9639-9641.

17 Y. Sun, D. Zhao, S. Fan and L. Duan, Sens. Actuator, B, 2015, 208, 512-517.

18 Y. Chen, C. Zhu, Z. Yang, J. Chen, Y. He, Y. Jiao, W. He, L. Qiu, J. Cen and Z. Guo, Angew. Chem., Int. Ed., 2013, 52, 1688-1691.

19 L. Meng, Y. Wu and T. Yi, Chem. Commun., 2014, 50, 48434845.

20 J. Li, C. Ji, W. Yang and M. Yin, Analyst, 2013, 138, 72897293.

21 Y.-Y. Li, M. Zhang, L.-F. Lu, A. Zhu, F. Xia, T. Zhou and G. Shi, Analyst, 2015, 140, 6108-6113.

22 G. S. Loving, M. Sainlos and B. Imperiali, Trends Biotechnol., 2010, 28, 73-83.

23 M. E. Vazquez, J. B. Blanco and B. Imperiali, J. Am. Chem. Soc., 2005, 127, 1300-1306.

24 I. Grabchev, I. Moneva, V. Bojinov and S. Guittonneau, J. Mater. Chem., 2000, 10, 1291-1296.

25 K. Baathulaa, Y. Xu and X. Qian, J. Photochem. Photobiol., A, 2010, 216, 24-34.

26 R. M. Duke, E. B. Veale, F. M. Pfeffer, P. E. Kruger and T. Gunnlaugsson, Chem. Soc. Rev., 2010, 39, 3936-3953.

27 S. Mallick and K. Pal, J. Colloid Interface Sci., 2015, DOI: 10.1016/j.jcis.2015.1012.1022.

28 M. E. Vazquez, D. M. Rothman and B. Imperiali, Org. Biomol. Chem., 2004, 2, 1965-1966.

29 T. Kogure, H. Kawano, Y. Abe and A. Miyawaki, Methods, 2008, 45, 223-226.

30 I. Saito, M. Takayama and S. Kawanishi, J. Am. Chem. Soc., 1995, 117, 5590-5591.

31 Z. Z. Li, C. G. Niu, G. M. Zeng, Y. G. Liu, P. F. Gao, G. H. Huang and Y. A. Mao, Sens. Actuators B, 2006, 114, 308-315.

32 P. Nandhikonda and M. D. Heagy, Org. Lett., 2010, 12, 4796-4799. 
33 M. Sainlos and B. Imperiali, Nat. Protoc., 2007, 2, 32193225.

34 M. Eugenio Vazquez, D. M. Rothman and B. Imperiali, Org. Biomol. Chem., 2004, 2, 1965-1966.
35 G. Loving and B. Imperiali, J. Am. Chem. Soc., 2008, 130, 13630-13638.

36 K. Baathulaa, Y. Xu and X. Qian, Nat. Protoc., 2011, 6, 1990-1997. 